\title{
Comparación de tres programas de prevención escolar para reducir el consumo de cannabis, en función del tipo de aplicador*
}

\section{Comparison of three school-based prevention programs to reduce cannabis use as a function of provider type}

\author{
Mónica Gázquez Pertusa \\ Universidad Miguel Hernández, España \\ José Antonio García del Castillo Rodríguez ${ }^{* *}$ \\ Universidad Miguel Hernández, España \\ Álvaro García del Castillo-López \\ Universidad Miguel Hernández, España \\ Carmen López-Sánchez \\ Universidad de Alicante, España
}

\footnotetext{
**Instituto de Investigación de Drogodependencias (INID). Correo electrónico: garciadelcastillo.rodriguez@gmail.com
}

Recepción: 04 Febrero 2014 | Aprobación: 22 Septiembre 2016

\section{RESUMEN}

El propósito general de este trabajo se encuadra en la línea de investigaciones que pretenden elevar los niveles de calidad de las estrategias preventivas en el consumo de cannabis, analizando la eficacia diferencial de los programas escolares al variar el perfil de las personas encargadas de implementarlos. Para ello, compara los resultados de la aplicación de tres programas de prevención escolar sobre el consumo de cannabis y las actitudes hacia su consumo, en función del tipo de aplicador empleado. La muestra se compuso de 280 alumnos de $1^{\circ}$ de Educación Secundaria Obligatoria (ESO), distribuidos en siete condiciones experimentales: seis de tratamiento (grupos experimentales) y otra en lista de espera (grupo control). Los grupos experimentales estuvieron determinados por la existencia de dos factores: Tipo de programa (programa Construyendo Salud, programa Saluda, programa Barbacana y Grupo Control) y tipo de Agente de aplicación (profesor habitual o psicólogo externo). Los profesores obtienen mejores resultados cuando aplican los programas Barbacana y Saluda, y los psicólogos cuando aplican el programa Construyendo Salud, tanto en el consumo de cannabis como en las actitudes relacionadas. Los resultados hallados apuntan hacia un efecto de interacción entre el tipo de programa y el tipo de aplicador.

Palabras clave

cannabis, prevención escolar, evaluación de programas, agentes de aplicación, eficacia.

\begin{abstract}
The aim of this work is fitted in line with research that intended to raise levels of quality of preventive strategies developed, analyzing the differential effectiveness of the school-based programs depending on the application agent. This study analyses the effects of three schoolbased prevention programs on cannabis use and attitudes towards drugs use as a function of provider type. Two hundred eighty students from the first year of Secondary Education were distributed among seven experimental conditions: six treatment conditions (experimental groups) and waiting list condition (control group). Experimental groups were determined by two factors: Type of program (Construyendo Salud program, Saluda program, Barbacana program and Control Group) and
\end{abstract}


Type of provider (regular teacher or external psychologist). The teachers applying the Barbacana and Saluda programs and psychologists applying the Construyendo Salud program obtain better outcomes in both cannabis use and attitudes. The results point out an interaction effect between type of program and type of applicators.

Keywords

cannabis, school prevention, program evaluation, application agents, efficacy.

Para citar este artículo : García del Castillo, J.A. (2016). Comparación de tres programas de prevención escolar para reducir el consumo de cannabis, en función del tipo de aplicador. Universitas Psychologica, 15 (4). http://dx.doi.o rg/10.11144/Javeriana.upsy15-4.cppe

\section{Introducción}

El consumo de cannabis inicialmente estuvo asociado a clases sociales marginales. Sin embargo, a partir de los años sesenta y setenta su uso aumentó progresivamente de forma paralela a la cultura hippie (Merino, 2000). Durante la década de los noventa su consumo se generalizó prácticamente en todos los países desarrollados, adquiriendo una imagen social de peligrosidad equivalente a la del tabaco y el alcohol. En la actualidad está ampliamente evidenciado que el consumo de cannabis provoca importantes consecuencias negativas sobre la salud (Ashton, 2002; Bobes \& Calafat, 2000; Guxens, Nebot, Ariza, \& Ochoa, 2007; Kalant, 2004), constituyéndose incluso como un factor de riesgo para el desarrollo de trastornos como la esquizofrenia (Parolaro, 2010) en sujetos vulnerables (Arias, 2007). No obstante, y pese a la evidencia empírica disponible, el cannabis es la sustancia ilegal más consumida (Observatorio Español sobre Drogas, 2010; Observatorio Europeo de las Drogas y Toxicomanías, 2010), oscilando el número de consumidores entre 142.6 y 190.3 millones de la población mundial de 15 a 64 años (Oficina de las Naciones Unidas contra la Droga y el Delito, 2010). En Europa, actualmente, las cifras nos indican que la tendencia al alza comienza a estabilizarse mostrando pautas descendentes en algunos países, aunque todavía permanece a niveles máximos históricos (OEDT, 2010).

Con respecto a las estrategias preventivas, los datos disponibles no justifican la aplicación de programas que se centren en prevenir el consumo de cannabis sin abordar el uso de otras drogas, especialmente las drogas legales (Amengual, 2000). Por tanto, la gran mayoría de intervenciones desarrolladas se han centrado en implementar programas aplicados principalmente al ámbito escolar, dirigidos a prevenir el consumo de las llamadas drogas de acceso o puerta de entrada al uso de otras drogas (Bobes, Bascarán, González, \& Sáiz, 2000). En cuanto a su eficacia, los estudios de revisión sugieren que los programas que consiguen reducir el consumo de cannabis y otras drogas ilegales son aquellos que utilizan una metodología interactiva (Tobler et al., 2000) y combinan varias intervenciones pertenecientes al modelo de influencia social (Skara \& Sussman, 2003), incluyendo principalmente el entrenamiento de habilidades junto con componentes informativos (Lemstra et al., 2010; Faggiano et al., 2008, 2010).

Sin embargo, pese a lo anteriormente mencionado, no todas las intervenciones basadas en el modelo de influencia social son igualmente eficaces (Cuijpers, 2002). Por ello, actualmente, las investigaciones que examinan los componentes específicos relacionados con la efectividad de estas intervenciones están aumentando de forma considerable.

Un ejemplo de ello lo forman los estudios que examinan la eficacia diferencial de una misma intervención al variar el perfil del agente que la aplica. A este respecto, aunque tradicionalmente los programas escolares para prevenir el consumo de drogas han sido aplicados por los profesores de los centros escolares (St. Pierre, Osgood, Siennick, Kauh, \& Burden, 2007), muchos de los programas han evaluado su eficacia empleando una amplia gama de agentes externos (profesores-tutores, profesionales de la salud, especialistas en prevención, pares, personal de investigación, etc.). 
Un tópico que en la actualidad continúa generando controversia es el referente a qué tipo de monitor es más idóneo para llevar a cabo la prevención escolar (Gázquez, García del Castillo, \& Espada, 2010; Macià, Olivares, \& Méndez, 1993). A este respecto, diversas investigaciones han intentado dilucidar esta cuestión mostrando unos resultados un tanto dispares. En algunos estudios son los expertos ajenos al centro quienes obtienen mejores resultados (Calafat, Amengual, Farres, \& Borrás, 1984; Cameron et al., 1999; Espada, Rosa, \& Méndez, 2003; García, Fernández, Carrillo \& Sánchez, 2006; Nozu et al., 2006; Tobler et al.,2000). Por el contrario, otros autores han hallado resultados más favorables con los profesores habituales del aula (Alonso \& Del Barrio, 1997; 1998; Bruvold, 1993; Fernández, Nebot, \& Jané, 2002; Harrington, Giles, Hoyle, Feeney, \& Yungbluth, 2001; McNeal, Hansen, Harrington, \& Giles, 2004). Finalmente, otros estudios no han mostrado diferencias en función del tipo de aplicador (Gómez, Luengo, \& Romero, 2002; Rohrbach, Dent, Skara, Sun, \& Sussman, 2007; Skara, Rohrbach, Sung, \& Sussman, 2005; Sun, Sussman, Dent, \& Rohrbarch, 2008; White, Buckley, \& Hassan, 2004).

Pese a la heterogeneidad que muestra la evidencia acumulada, la literatura especializada destaca la influencia que ejerce la variable tipo de aplicador al moderar los efectos de las intervenciones escolares (Gázquez et al., 2010; Gottfredson \& Wilson, 2003; Hansen, 1992; Mellanby, Rees, \& Tripp, 2000).

En este trabajo se describe la evaluación de los efectos de tres intervenciones de prevención escolar sobre el consumo de cannabis y, a su vez, se analiza el efecto moderador que la variable tipo de aplicador ejerce sobre la eficacia de las tres intervenciones. Para ello, se comparan los resultados de tres programas para prevenir el consumo de drogas: El programa Construyendo Salud de Luengo, Gómez, Garra, Romero, y Otero (1998), el programa Saluda de Espada y Méndez (2003) y el programa Barbacana de García-Rodríguez y López-Sánchez (1998), según fueran aplicados por los profesores de los centros escolares o por psicólogos externos, con respecto a un grupo que permanece en lista de espera.

\section{Método}

\section{Participantes}

Un total de siete centros públicos de educación secundaria de la ciudad de Elche (Alicante, España), estuvieron dispuestos a participar en el estudio. La muestra estuvo compuesta por 280 adolescentes (50\% varones y 50\% mujeres) matriculados en primero de Educación Secundaria Obligatoria (ESO), con una edad media igual a $12.42(D T=0.57)$.

$\mathrm{Al}$ analizar las características sociofamiliares, se destaca que el estado civil de los progenitores mayoritariamente corresponde a la categoría casados (83\%), un $13 \%$ a la categoría separados, un $2 \%$ viudos y a la categoría en pareja le corresponde un $2 \%$. En lo referente al nivel cultural de ambos progenitores, alrededor de un $40 \%$ poseen estudios elementales y aproximadamente un 4.5-5\% estudios universitarios superiores.

Con referencia a las relaciones familiares, un $62.5 \%$ de los sujetos las cataloga como muy buenas, seguido de un $33.4 \%$ que las clasifica como buenas. Un $2.2 \%$ describe sus relaciones como indiferentes $\mathrm{y}$, tan solo, un $0.9 \%$ del total de la muestra las señala como malas o muy malas. Los resultados obtenidos en los ítems relacionados con la percepción de pautas familiares en cuanto al rendimiento escolar, el cumplimiento de normas y la permisividad ante el consumo de alcohol o tabaco se muestran en la Tabla 1. 
TABLA 1

Percepción de las pautas familiares

\begin{tabular}{lccccc}
\hline & Nada & Algo & Regular & Bastante & Mucho \\
\hline RE & $1 \%$ & $1 \%$ & $1 \%$ & $28 \%$ & $69 \%$ \\
SP & $25 \%$ & $23 \%$ & $23 \%$ & $21 \%$ & $8 \%$ \\
PC & $1 \%$ & $2 \%$ & $3 \%$ & $16 \%$ & $76 \%$ \\
\hline
\end{tabular}

Fuente: elaboración propia

Con respecto a las relaciones en el aula, en torno a un $84 \%$ de los sujetos refieren sentirse bien o muy bien con los compañeros, un $13 \%$ declaran sentirse regular y un $3 \%$ de sujetos afirma sentirse mal o muy mal.

En lo referente al rendimiento académico, la media de las asignaturas suspendidas en la última evaluación se sitúa en torno a 2.5. Siendo 13 el número mayor de asignaturas suspendidas (0.9\%).

Con respecto al dinero disponible semanalmente, la media se sitúa en torno a los $6.5 €$. No obstante, cabe destacar que las cantidades recibidas oscilan entre 0 y 50 euros semanales. La media de dinero gastado a la semana corresponde a $4.9 €$

Cuando se analiza el consumo de las diversas sustancias se constata que del total de la muestra un $10.4 \%$ afirma haber probado el tabaco, un $14 \%$ el alcohol y un $5.7 \%$ el cannabis. En la Tabla 2 se detallan los porcentajes de sujetos que declaran consumir otras sustancias psicoactivas. Los resultados sobre el consumo de sustancias nos muestran que los niveles son lo suficientemente bajos para poder realizar una intervención preventiva universal del consumo de drogas.
TABLA 2

Incidencia consumo de otras sustancias psicoactivas

\begin{tabular}{lcc}
\hline & SI & NO \\
\hline Estimulantes & $1.8 \%$ & 98.2 \\
Tranquilizantes & 3.6 & 96.4 \\
Analgésicos & 8.2 & 91.8 \\
Cocaína & $0.4 \%$ & $99.6 \%$ \\
Heroína & 0 & $100 \%$ \\
LSD & $0.4 \%$ & $99.6 \%$ \\
Disolventes & 0 & $100 \%$ \\
\hline
\end{tabular}

Fuente: elaboración propia

\section{Diseño}

Se utilizó un diseño cuasi-experimental de grupo control no equivalente, con mediciones pretest, postest y seguimiento (transcurridos veinticuatro meses de la aplicación de los programas).

Los centros fueron asignados aleatoriamente a siete condiciones experimentales: seis de intervención (grupos experimentales) y otra en lista de espera (grupo control). Los grupos experimentales estuvieron determinados por la existencia de dos factores: Tipo de programa: programa Construyendo Salud (CS), programa Saluda (SLD), programa Barbacana (BRC) y Grupo Control (GC), y tipo de Agente de aplicación : profesor habitual o psicólogo externo. Se establecieron como criterios de inclusión que los sujetos fueran escolares matriculados en $1^{\circ}$ de la ESO y el compromiso por parte de los centros a no desarrollar ninguna intervención preventiva del consumo de drogas durante el período de aplicación y seguimiento de los programas (24 meses). Los tamaños muestrales se detallan en la Tabla 3. 
Tabla 3

Tamaños muestrales en los tres momentos temporales de evaluación.

\begin{tabular}{|c|c|c|c|c|c|c|c|c|}
\hline & \multicolumn{2}{|c|}{ C. SALUD } & \multicolumn{2}{|c|}{ SALUDA } & \multicolumn{2}{|c|}{ BARBACANA } & \multirow{2}{*}{ G.C. } & \multirow{2}{*}{ Total } \\
\hline & Prof. & Psi. & Prof. & Psi. & Prof. & Psi. & & \\
\hline Pretest (T1) & 40 & 40 & 40 & 40 & 40 & 40 & 40 & 280 \\
\hline Postest (T2) & 38 & 40 & 39 & 39 & 40 & 40 & 38 & 274 \\
\hline Seguimt.(T3) & 37 & 38 & 37 & 39 & 40 & 38 & 38 & 270 \\
\hline
\end{tabular}

Prof $=$ Profesores; Psi $=$

Psicólogos; GC = Grupo Control.

Fuente: elaboración propia.

\section{Instrumentos}

Para valorar la eficacia de las intervenciones aplicadas se emplearon los siguientes cuestionarios:

Cuestionario de Consumo de Sustancias (COTAM) de García-Rodríguez y LópezSánchez (1994). Se trata de un cuestionario descriptivo que consta de 10 ítems exploratorios del consumo de tabaco, alcohol y cannabis a través de diversos indicadores, con el objetivo de conocer las frecuencias de consumo, tanto en cantidad como en periodicidad. Por una parte, se explora la incidencia del consumo de tabaco, alcohol y cannabis, con objeto de sondear el porcentaje de sujetos que declaran haber probado alguna de estas sustancias, con independencia del tipo de consumo que realicen (esporádico, regular, habitual, etc). Y por otra, la frecuencia mensual del consumo de tabaco y alcohol, la cantidad de cigarrillos fumados a diario y el porcentaje de sujetos que declara haber experimentado como mínimo un episodio de embriaguez. Estos últimos indicadores se han seleccionado con la finalidad de poder estimar la intensidad del consumo de tabaco y alcohol. Este cuestionario incluye varios índices de consumo con objeto de detectar posibles sesgos de respuesta.

Escala de Actitudes hacia las drogas ilegales (ESACDRO) de García-Rodríguez y LópezSánchez, (2001) . Compuesta de 13 ítems con formato de respuesta tipo Likert (1-5), evalúa actitudes y predisposiciones hacia el consumo de drogas ilegales. Se desglosa en tres factores, cuyos ítems muestran saturaciones mayores a 0.40 y un total de la varianza explicada de 59.33\%. Factor 1: Disposición actitudinal desfavorable al consumo de drogas ilegales; Factor 2: Percepción de desagrado; y Factor 3: Percepción de riesgo. Una puntuación elevada en cada uno de los factores indica una actitud contraria al consumo de drogas. La escala muestra correlaciones test-retest significativas $\mathrm{r}=0.437, \mathrm{p} \leq .01$ (García-Rodríguez \& LópezSánchez, 2001).

\section{Programas de prevención}

Se aplicaron tres programas de prevención del consumo de drogas, enmarcados en la tipología de Mejora de las Competencias Personales, cuya eficacia se ha contrastado de forma independiente con población escolar española.

El programa Construyendo Salud (CS) de Luengo et al. (1998) es una ampliación y adaptación a nuestro país del programa Life Skills Training (Botvin \& Tortu, 1988). Estructurado en 17 sesiones aplicadas de forma interactiva, se compone de siete ejes principales: información, autoestima, toma de decisiones, control emocional, habilidades sociales, comportamiento prosocial y ocio. Junto con estos componentes se incluyen técnicas cognitivoconductuales: instrucciones, modelado, ensayo conductual, retroalimentación, reforzamiento y reestructuración cognitiva.

La aplicación del programa se ha evaluado a través de diversos estudios mostrando su eficacia para reducir el consumo de tabaco y alcohol, aumentar el grado de información y reducir las actitudes favorables al tabaco y alcohol (Luengo, Romero, Gómez-Fragüela, Guerra, \& Lence, 1999). También se ha mostrado eficaz para reducir la frecuencia del consumo de tabaco, cerveza y cannabis cuando es aplicado por técnicos y para reducir el consumo de licores cuando es aplicado por profesores (Gómez et al., 2002).

El programa Saluda (SLD) de Espada y Méndez (2003) se centra en prevenir el abuso de alcohol y drogas de síntesis durante el fin de semana. A lo largo de diez 
sesiones de idéntica estructura y mediante una metodología interactiva trata de generar actitudes contrarias al consumo de drogas y enseñar estrategias cognitivo-conductuales para afrontar situaciones relacionadas con su consumo. Está formado por cuatro componentes: actividades de ocio saludable, entrenamiento en resolución de problemas, en habilidades sociales y en habilidades de resistencia a la presión. Incluye, además, un módulo de información sobre drogas, el establecimiento público sobre la decisión de no consumir y el entrenamiento en autorefuerzo. Con respecto a su evaluación, el programa se ha mostrado eficaz para reducir el consumo de alcohol (Pereira \& GarcíaFernández, 2009), aumentar el nivel objetivo sobre el consumo de alcohol y drogas de síntesis y las actitudes desfavorables hacia el consumo (Espada, Orgilés, Méndez, García-Fernández, \& Inglés, 2008).

Por último, el programa Barbacana (BRC) de García-Rodríguez y López-Sánchez (1998) se desarrolla en ocho sesiones que mantienen una estructura común y pretende fomentar una actitud favorable al no consumo y facilitar la toma de decisiones racionales ante el uso de drogas. Para ello, utiliza técnicas de modelado y ensayo de conducta (videos, role-playing), entrenamiento en resolución de problemas y técnicas de transmisión de información interactivas. En cuanto a su evaluación, el programa ha mostrado su eficacia para aumentar el nivel objetivo de información sobre las drogas, retrasar el inicio del consumo de sustancias ilegales y disminuir la incidencia del consumo de tabaco y alcohol (García del Castillo, López, Fernández, \& Catalá, 2003).

\section{Procedimiento}

Antes de iniciar la implementación de los programas todos los agentes de aplicación recibieron un curso en vivo de 20 horas de duración, sobre los fundamentos de la prevención escolar y la aplicación de las sesiones, en función del programa asignado a cada aplicador.
El protocolo del estudio fue aprobado por la Comisión de Ética en la Investigación Experimental de la Universidad Miguel Hernández. Todos los padres y/o tutores de los participantes dieron su consentimiento informado por escrito después de recibir una descripción detallada del estudio. Tras obtener los permisos pertinentes se procedió a aplicar las pruebas de evaluación. Todas las mediciones se llevaron a cabo de forma autoaplicada y anónima, dentro del horario escolar. Para facilitar el seguimiento de los cuestionarios en las tres evaluaciones se solicitó a los sujetos que elaboraran una clave fácil de recordar.

Para llevar a cabo el estudio se ofertó la aplicación de programas de prevención del consumo de drogas a todos los centros públicos de educación secundaria de la localidad de Elche (Alicante, España). Se establecieron como criterios de inclusión que los sujetos fueran escolares matriculados en primero de Educación Secundaria Obligatoria (ESO) y el compromiso por parte de los centros a no desarrollar ninguna intervención preventiva del consumo de drogas durante el periodo de aplicación y seguimiento de los programas. Del total de centros a los que se les ofertó la aplicación de los programas, siete estuvieron dispuestos a participar. Estos siete centros fueron asignados a las distintas condiciones experimentales aleatoriamente, seis centros a los grupos experimentales y uno como grupo control-lista de espera. De esta manera cada programa fue aplicado en dos centros distintos que, a su vez, se diferenciaban entre sí por las personas encargadas de impartirlos, docentes y psicólogos, asignados al azar a cada grupo experimental. Las mediciones del postest se realizaron justo a la finalización de la aplicación de cada programa y las mediciones de seguimiento transcurridos 24 meses desde la aplicación del postest.

\section{Análisis de los datos}

Los resultados se analizaron con el software PASW Statistics-18. Para las variables cuantitativas, dado que los datos de la muestra 
presentaron heterocedasticidad y no siguieron una distribución normal, se ha empleado el estadístico $T$ de Student intragrupo e intergrupos, ajustando la tasa de error por comparaciones mediante la corrección Bonferroni, dividiendo el alfa nominal entre el número de comparaciones a efectuar. Para cuantificar la magnitud del efecto se calculó la diferencia de medias estandarizada mediante la $d$ de Cohen. Los datos sobre el consumo de cannabis se han analizado mediante la prueba $Q$ de Cochran (intragrupos) y $\chi^{2}$ de Pearson para proporciones independientes. Para calcular el tamaño del efecto se obtuvieron las Odds Ratio, a partir de los porcentajes de sujetos no consumidores transformándolas a continuación en el índice $\mathrm{d}_{\mathrm{Cox}}$ (Sánchez-Meca, Marín-Martínez, \& Chacón-Moscoso, 2003). Se utilizó un nivel de confianza del $95 \%$ para todos los análisis efectuados.

\section{Resultados}

Los estadísticos descriptivos para cada condición experimental se detallan en la Tabla 4. La homogeneidad de los grupos experimentales y control fue comprobada antes de la aplicación de los programas. Los grupos fueron equivalentes en la edad ( $F=2.63 ; p=0.853)$, en el sexo $\left(\chi^{2}\right.$ $=11,4 ; p=0.77)$, en el consumo de cannabis $\left(\chi^{2}=1.59 ; p=0.953\right)$ y en las actitudes hacia el consumo de drogas ilegales.

\section{Experimentación del consumo de cannabis}

\section{TABLA 4}

Estadísticos descriptivos en la evaluación inicial

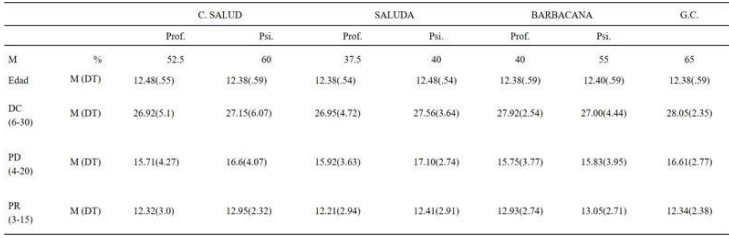

$$
\begin{aligned}
& \text { M = Mujeres } ; \mathrm{DC}=\text { Disposición } \\
& \text { Consumo; Percepción Desagrado; PR } \\
& =\text { Percepción Riesgo Prof }=\text { Profesores; } \\
& \text { Psi = Psicólogos; GC = Grupo Control. } \\
& \text { Fuente: elaboración propia. }
\end{aligned}
$$

Cuando se analiza el porcentaje de sujetos que declaran haber consumido cannabis alguna vez en la vida se observan diferencias relevantes entre los grupos experimentales y control, tanto

\begin{tabular}{|c|c|c|c|c|c|c|c|c|}
\hline \multicolumn{2}{|c|}{$\begin{array}{l}\text { Condición } \\
\text { EFrerimentat }\end{array}$} & \multicolumn{2}{|c|}{ PRETEST \% } & \multicolumn{2}{|c|}{ POSTEST \% } & \multicolumn{2}{|c|}{ SEGUIM. $\%$} & \multirow[t]{2}{*}{ Q de Cochra } \\
\hline & & S1 & No & SI & No & SI & No & \\
\hline \multirow[t]{2}{*}{ cs } & Prof. & 7.5 & 92.5 & 7.9 & 92.1 & 10.8 & 89.2 & 1 \\
\hline & Psi. & 5 & 95 & 2.5 & 97.5 & 5.3 & 94.7 & 0.667 \\
\hline \multirow[t]{2}{*}{ sL. } & Prof. & 2.5 & 97.5 & 2.6 & 97.4 & 0 & 100 & 2 \\
\hline & Psi & 5 & 90 & 2.6 & 97.4 & 0 & 100 & 3 \\
\hline \multirow[t]{2}{*}{ BRC } & Prof. & 5 & 90 & 0 & 100 & 5 & 95 & 4 \\
\hline & Psic. & 7.5 & 92.5 & 0 & 100 & 7.9 & 92.1 & 4.5 \\
\hline G.C. & & 7.5 & 92.5 & 13.2 & 86.8 & 18.4 & 81.6 & 4 \\
\hline
\end{tabular}
en el postest como en el seguimiento (ver Tabla $5)$.

\section{TABLA 5}

Consumo de cannabis

$$
\begin{gathered}
\text { Prof }=\text { Profesores } ; \text { Psi }=\text { Psicólogos } ; \text { CS } \\
=\text { Construyendo Salud } ; \text { SLD = Saluda; } \\
\text { BRC = Barbacana; G.C. }=\text { Grupo Control. } \\
* \text { p }<0.05 \\
\text { Fuente: elaboración propia. }
\end{gathered}
$$

Al profundizar en este examen, las comparaciones por pares muestran que en el postest son los grupos que recibieron el programa $\mathrm{BCR}$, en sus dos modalidades, los que arrojan menores porcentajes de experimentación con el cannabis al compararse con el grupo en lista de espera. En ambas comparaciones se obtiene un tamaño del efecto elevado (BCR-Profesores: $\mathrm{d}_{\mathrm{Cox}}=1.57$ y BCR-Psicólogos: $\left.\mathrm{d}_{\mathrm{Cox}}=1.54\right)$. La magnitud de las diferencias cuando se compara el grupo control con el resto de intervenciones es elevada para el programa SLD en sus dos modalidades $\left(\mathrm{d}_{\mathrm{Cox}}=0.87\right)$ y para el programa 
CS aplicado por psicólogos $\left(\mathrm{d}_{\mathrm{Cox}}=0.89\right)$, y moderada para el programa CS aplicado por profesores $(\mathrm{dCox}=0.31)$.

En el seguimiento, se observa que es el programa SLD en sus dos modalidades el que muestra menores porcentajes de sujetos consumidores comparado con el grupo control (SLD-Profesores: $\mathrm{d}_{\mathrm{Cox}}=1.75$ y SLD-Psicólogos: $\mathrm{d}_{\mathrm{Cox}}=1.78$ ). La magnitud del efecto en el resto de grupos experimentales es moderada para las condiciones BRC-Profesor $\left(\mathrm{d}_{\mathrm{Cox}}=0.79\right)$, CSPsicólogo $\left(\mathrm{d}_{\mathrm{Cox}}=0.75\right)$ y BCR-Psicólogo $\left(\mathrm{d}_{\mathrm{Cox}}\right.$ $=0.53$ ) y pequeña para el programa CS aplicado por los profesores $\left(\mathrm{d}_{\mathrm{Cox}}=0.35\right)$.

\section{Actitudes hacia las drogas ilegales}

En la Tabla 6 se presentan los estadísticos descriptivos relacionados con las actitudes en el postest y en el seguimiento, junto con las comparaciones intragrupos (diferencias entre evaluación inicial-postest y evaluación inicialseguimiento).
TABLA 6

Evolución temporal de las variables mediadoras. Comparaciones intragrupos

\begin{tabular}{|c|c|c|c|c|c|c|c|c|c|}
\hline \multirow[t]{2}{*}{$\begin{array}{l}\text { Variable } \\
\text { (rango) }\end{array}$} & & \multicolumn{2}{|c|}{ POSTEST (T2) } & \multicolumn{2}{|c|}{ SEGUIM. (T3) } & \multicolumn{2}{|c|}{$\begin{array}{l}\text { PRETEST- } \\
\text { POSTEST } \\
\text { (T1-T2) }\end{array}$} & \multicolumn{2}{|c|}{$\begin{array}{l}\text { PRETEST- } \\
\text { SEGUM. } \\
\text { (T1-T3) }\end{array}$} \\
\hline & & $\mathrm{M}$ & DT & $\mathrm{M}$ & DT & $T$ & |d & $T$ & d d \\
\hline \multirow{7}{*}{$\begin{array}{l}\text { Disposición } \\
\text { consumo }\end{array}$} & G.1 & 28.4 & (2.93) & 28.46 & (2.66) & -1.724 & 0.28 & -1.794 & 0.29 \\
\hline & G. 2 & 29.05 & (1.36) & 28.97 & (1.66) & -1.954 & 0.31 & -1.951 & 0.32 \\
\hline & G. 3 & 29.35 & (1.54) & 29.27 & 1.85) & $-3.336^{*}$ & 0.53 & $-3.097^{*}$ & 0.51 \\
\hline & G. 4 & 28.41 & (2.68) & 27.76 & (3.31) & -1.580 & 0.25 & -0.311 & 0.05 \\
\hline & G.5 & 28.98 & (1.69) & 29.05 & (1.96) & $-2.572^{*}$ & 0.41 & -2.232 & 0.35 \\
\hline & G.6 & 27.15 & (4.4) & 27.92 & (2.91) & -0.195 & 0.03 & -1.269 & 0.21 \\
\hline & G.7 & 27.58 & (2.09) & 27.08 & (2.65) & 1.639 & 0.27 & 2.084 & 0.34 \\
\hline \multirow{6}{*}{$\begin{array}{l}\text { Percepción } \\
\text { desagrado }\end{array}$} & G.1 & 17.3 & (3.07) & 17.40 & 2.31) & -1.932 & 0.31 & -2.219 & 0.36 \\
\hline & G.2 & 18.35 & (1.81) & 17.18 & 2.88) & $-2.696^{\circ}$ & 0.43 & -0.943 & 0.15 \\
\hline & G. 3 & 17.26 & (2.97) & 17.43 & (2.73) & -1.819 & 0.29 & -1.866 & 0.31 \\
\hline & G.4 & 16.78 & (3.02) & 17.10 & (3.39) & 0.496 & 0.08 & -0.000 & 0 \\
\hline & G.5 & 17.9 & (2.54) & 17.75 & (2.95) & $-3.446^{*}$ & 0.54 & $-2.900^{*}$ & 0.46 \\
\hline & G.6 & 16.73 & (3.33) & 16.89 & (2.96) & -1.607 & 0.25 & -1.412 & 0.23 \\
\hline \multirow{8}{*}{$\begin{array}{l}\text { Percepción } \\
\text { riesgo }\end{array}$} & G.7 & 15.63 & (2.78) & 15.10 & (3.11) & 2.013 & 0.33 & $2.558^{*}$ & 0.41 \\
\hline & G.1 & 13.71 & (1.5) & 13.67 & (1.67) & $-2.531^{*}$ & 0.41 & $-2.678^{*}$ & 0.44 \\
\hline & G.2 & 14.03 & (1.29) & 13.02 & (2.08) & $-2.911^{\circ}$ & 0.46 & -0.454 & 0.07 \\
\hline & G. 3 & 12.92 & (2.09) & 13.35 & (1.60) & -1.453 & 0.23 & -1.829 & 0.30 \\
\hline & G.4 & 12.28 & (2.66) & 12.56 & (2.87) & 0.286 & 0.05 & -0.274 & 0.04 \\
\hline & G.5 & 13.38 & (1.96) & 13.50 & (2.01) & -0.917 & 0.14 & -1.052 & 0.17 \\
\hline & G.6 & 12.18 & (2.91) & 11.95 & (2.94) & 1.706 & 0.27 & 2.036 & 0.33 \\
\hline & G.7 & 11.89 & (1.88) & 11.29 & (2.31) & 1.122 & 0.18 & $2.351^{*}$ & 0.38 \\
\hline
\end{tabular}

G.1 = CS-Profesor; G.2 = CS-Psicólogo; G. 3 = SLD-Profesor; G.4 = SLD-

Psicólogo; G.5 = BRC-Profesor; G.6

= BRC-Psicólogo; G.7 = G. Control. $* \mathrm{p}<0.025$

Fuente: elaboración propia.

En el postest muestran una mayor disposición desfavorable hacia el consumo de drogas los grupos dirigidos por los profesores cuando aplican los programas SLD $(d=0.53)$ y BRC ( $d$ $=0.41)$. Por otro lado, la percepción de desagrado aumenta en las condiciones CS-Psicólogo $(d=$ 0.43 ) y BRC-Profesor ( $d=0.54)$ y la percepción de riesgo aumenta en los grupos que recibieron el programa CS en sus dos modalidades (Profesor $d=0.41$; Psicólogo $d=0.46$ ).

En el seguimiento, en la disposición desfavorable al consumo, los cambios se mantienen para la condición SLD-Profesor $(d=$ 0.51). La percepción de desagrado aumenta en la condición BCR-Profesor ( $d=0.46)$ y disminuye en el grupo control ( $d=0.41$ ). Y por último, se detecta un aumento de la percepción de riesgo en el grupo que recibió el programa CS aplicado por los profesores ( $d=0.44$ ) y una disminución del $\mathrm{GC}(d=0.38)$.

Cuando se comparan los promedios registrados en los grupos experimentales frente al grupo control se encuentran diferencias 
relevantes tanto a corto como a medio plazo ( comparaciones intergrupos ).

\section{TABLA 7}

Comparaciones intergrupos actitudes. Grupos experimentales frente al control

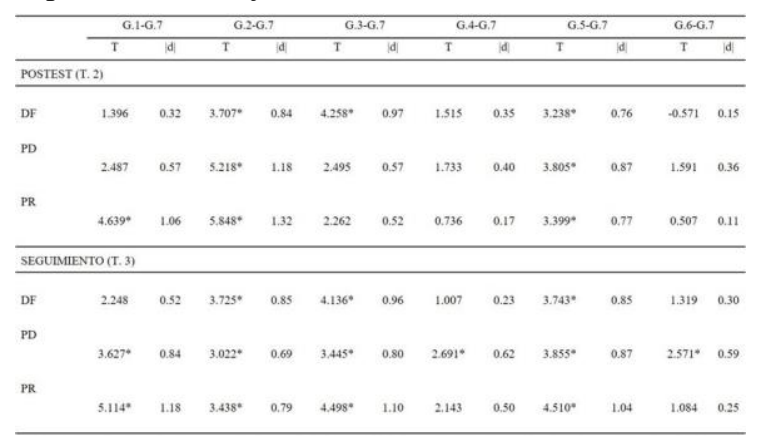

$\mathrm{DF}=$ Disposición Desfavorable; $\mathrm{PD}=$

Percepción Desagrado; $\mathrm{PR}=$ Percepción

Riesgo; G. 1 = CS-Profesor; G. 2 = CS-

Psicólogo; G.3 = SLD-Profesor; G.4 =

SLD-Psicólogo; G.5 = BRC-Profesor; G.6

= BRC-Psicólogo; G.7 = Grupo Control.

$* \mathrm{p}<0.008$

Fuente: elaboración propia.

Como puede observarse en la Tabla 7 , en el postest obtienen un promedio mayor que el GC en la disposición desfavorable al consumo, los profesores cuando aplican los programas SLD $(d$ $=0.97)$ y BCR $(d=0.76)$ y los psicólogos cuando aplican el programa CS $(d=0.84)$.

En la percepción de desagrado se detectan diferencias elevadas al comparar el grupo control con los grupos que recibieron el programa CS aplicado por psicólogos ( $d=1.18)$ y BRC aplicado por profesores ( $d=0.87$ ), a favor de los grupos experimentales. En lo que respecta a la percepción de riesgo, los grupos que recibieron el programa CS aplicado por ambos monitores (profesores: $d=1.06$ y psicólogos: $d=1.32$ ) y el programa BRC aplicado por los profesores ( $d=0.77$ ) muestran diferencias elevadas al compararse con el GC, a favor de los grupos experimentales.

Transcurridos veinticuatro meses de la finalización de los programas (seguimiento), en lo que respecta a la disposición actitudinal desfavorable hacia el consumo de drogas ilegales las diferencias registradas en el postest tienden a incrementarse. De nuevo es el grupo que recibe el programa SLD aplicado por los profesores el que obtiene una diferencia mayor ( $d=0.96$ ) con respecto al grupo control, seguido por las modalidades CS-psicólogos y BCR-profesores ( $d=0.85$ en ambos casos).

En cuanto a la percepción de desagrado, se observa la existencia de diferencias relevantes entre el grupo control con respecto a todos los grupos que recibieron alguna modalidad de intervención, aunque son los profesores los que obtienen tamaños del efecto mayores cuando aplican los tres programas (BRC $d=0.87$; CS $d$ $=0.84 ;$ SLD $d=0.80$ ).

Por último, en el factor orientado a la percepción de riesgo, las diferencias detectadas en el postest se mantienen alcanzando magnitudes elevadas. De nuevo son los profesores los que comparados con el grupo control logran aumentar de forma relevante la percepción de riesgo cuando aplican los tres programas, obteniendo tamaños del efecto elevados (CS $d=1.18 ;$ SLD $d=1.10 ;$ BRC $d=$ 1.04).

\section{Discusión}

El objetivo de este trabajo ha consistido en valorar la eficacia de tres programas escolares para prevenir el consumo de cannabis e incidir sobre las actitudes relacionadas con su uso según el tipo de aplicador. Para ello, se aplicaron de forma simultánea los programas empleando dos tipos de agentes: los profesores habituales del aula y psicólogos externos al centro. Por tanto, con objeto de valorar los efectos obtenidos al implementar las intervenciones se han efectuado comparaciones intragrupos y entre los grupos experimentales y control.

En referencia al consumo de cannabis a corto plazo (postest) todas las intervenciones desarrolladas consiguen reducir el porcentaje de sujetos que declara fumar cannabis, siendo el programa BRC aplicado por ambos monitores el que mayores tamaños de efecto obtiene. Sin embargo, a medio plazo (seguimiento) es el programa SLD en sus dos modalidades el 
que consigue lograr una diferenciación más importante con respecto al grupo control.

También se han obtenido cambios en las actitudes hacia el consumo de drogas ilegales en dirección a un fortalecimiento de las actitudes favorables al mantenimiento de la salud, manifestadas a través de creencias y expectativas asociadas a bajas inclinaciones para consumir. Este efecto se confirma mediante el análisis de la evolución de los promedios registrados a lo largo de la evaluación postintervención en los grupos experimentales comparados tanto con la evaluación inicial (pretest) como con la evolución registrada en el grupo control, en el que las actitudes y disposiciones permisivas hacia el consumo se han ido afianzando.

No obstante, y pese a que actitud y conducta se vinculan de forma compleja, este efecto atenuador de la tendencia natural registrada en el grupo sin intervención resulta relevante. A este respecto, aunque la esfera actitudinal no es el único determinante de la conducta manifiesta, las actitudes actúan como moduladoras de las experimentaciones e incluso de los propios hábitos de consumo (Moral, Rodríguez, Ovejero, \& Sirvent, 2009).

En síntesis, los resultados obtenidos en este estudio muestran que los programas son eficaces para reducir el porcentaje de sujetos que declaran fumar cannabis y para promover y consolidar actitudes contrarias al consumo de drogas. Estos resultados se sitúan en consonancia con los hallazgos encontrados en diversos estudios que indican que las intervenciones basadas en el entrenamiento en habilidades personales son eficaces para reducir el consumo de cannabis y las variables que lo median, a corto plazo (Faggiano, et al., 2008; Lemstra et al., 2010; Tobler et al., 2000), transcurridos dos (Sussman, Dent, Craig, Ritt-Olsen, \& McCuller, 2002; Sussman, Sun, McCuller, \& Dent, 2003) y hasta 5 años de la aplicación de las intervenciones (Furr-Holden, Ialongo, Anthony, Petras, \& Kellam, 2004).

Con respecto al tipo de programa más eficaz, el efecto de las intervenciones varía en función del tipo de monitor, el constructo analizado y el momento temporal en el que se toman las medidas (efectos a corto y a medio plazo). En líneas generales, los programas SLD y BRC logran tamaños del efecto mayores cuando son aplicados por el profesorado de los centros escolares, mientras que el programa CS obtiene mejores resultados cuando lo implementan los psicólogos externos. En este sentido, la variabilidad mostrada en nuestros resultados se sitúa en consonancia con los argumentos que defienden autores como Gottfredson y Wilson (2003) o Stead y Angus (2004) al afirmar que los efectos preventivos de un programa concreto pueden variar en función del tipo de agente encargado de aplicarlo, debido a un efecto de interacción entre el tipo de programa y el tipo de aplicador.

En cuanto al tipo de agente más apropiado, en general, podemos concluir que los profesores de los centros escolares son los agentes más eficientes considerando la relación costobeneficio. Puesto que la magnitud de las diferencias encontradas en algunos constructos a favor de las condiciones caracterizadas por la aplicación de los psicólogos externos, no es lo suficientemente importante para justificar el costo que generaría su intervención.

Aunque posiblemente, más que analizar cuál es el perfil idóneo para aplicar programas preventivos, la clave radique en qué características específicas o competencias ha de poseer un monitor para manejar eficazmente las técnicas que componen los elementos claves de los protocolos, y cómo y en qué medida el entrenamiento puede mejorar tales habilidades (Cuijpers, 2002; Gázquez et al., 2010).

Por último, entre las limitaciones del trabajo realizado cabe destacar el tamaño de la muestra que podría afectar a la potencia de los contrastes efectuados. Por tanto, en futuras investigaciones con objeto de aumentar la generalización de los resultados y corroborar los efectos hallados, se recomienda abarcar a poblaciones más numerosas y heterogéneas.

La evaluación a largo plazo es un aspecto a mejorar para determinar si las reducciones detectadas posteriormente se mantienen $o$, incluso, si se traducen en una no progresión hacia una implicación más seria en el consumo. 
En este sentido, tal y como indican Gómez et al. (2002), para evaluar la eficacia de un programa preventivo es necesario considerar que el consumo de drogas es un fenómeno dinámico, en el que los cambios producidos son distintos según el momento evolutivo de los sujetos.

De igual modo, se recomienda la incorporación de sesiones de recuerdo que refuercen los aprendizajes adquiridos tras la aplicación de los programas (McBride, Farringdon, Midford, \& Phillips, 2001; Shope, Elliott, Raghunathan, \& Waller, 2001; Skara \& Sussman, 2003).

\section{Referencias}

Alonso, C., \& Del Barrio, V. (1997). Efectividad de tres Intervenciones para la prevención del consumo de tabaco en el medio escolar. Revista de Psicología General y aplicada, 50 (2), 223-241.

Alonso, C., \& Del Barrio, V. (1998). Efectividad de tres Intervenciones para la prevención del consumo de alcohol en la escuela. Análisis y Modificación de Conducta, 24 (97), 679-701.

Amengual, M. (2000). Enfoques preventivos del uso y abuso de cannabis y problemas asociados. Adicciones, 12 (Supl.2), 281-300.

Arias, F. (2007). Revisión sobre el consumo de cannabis como factor de riesgo en la esquizofrenia. Adicciones, 19 (2), 191-204

Ashton, H. (2002). Cannabis or health? Current Opinion in Psychiatry, 15 (3), 247-253.

Bobes, J., Bascarán, M.T., González, M.P., \& Sáiz, P.A. (2000). Epidemiología del uso/ abuso de cannabis. Adicciones, 12 (Supl.2), 31-40.

Bobes, J., \& Calafat, A. (2000). De la Neurobiología a la psicosociología del uso-abuso del cannabis. Adicciones, 12 (Supl.2), 7-17.

Botvin, G., \& Tortu, S. (1988). Preventing adolescent substance abuse throught life skills training. En R. Price, E. Cowen, R. Lorion y J. Ramos-Mckay (Eds).
Fourten ounces of prevention (pp. 98-110). Washington: APA.

Bruvold, W.H. (1993). A meta-analysis of adolescent smoking prevention programs. American Journal of Public Health, 83, 872-880.

Calafat, A., Amengual, M., Farrés, C., \& Borrás, M. (1984). Eficacia de un programa de prevención sobre drogas, especialmente centrado en la "toma de decisiones", según sea desarrollado por especialistas o por profesores de los alumnos. Drogalcohol, 9 (3), 147-170.

Cameron, R., Brown, K.S., Best, J. A., Pelkman, C. L., Madill, C.L., Manske, S. R., \& Payne, E. (1999). Effectiveness of a Social Influences Smoking Prevention Program as a Function of Provider Type, Training Method, and School Risk. American Journal of Public Health, 89 (12), 18271831.

Cuijpers, P. (2002). Effective ingredients of school-based drug prevention programs. A systematic review. Addictive Behaviors, 27 (6), 1009-1023.

Espada, J. P., \& Méndez, F. X. (2003). Prevención del abuso de alcohol y drogas de síntesis . Madrid: Ediciones Pirámide.

Espada, J.P., Orgilés, M., Méndez, F.X., GarcíaFernández, J.M., \& Inglés, C. (2008). Efectos del programa Saluda sobre factores cognitivos relacionados con el consumo de drogas. Salud y drogas, 8 (1), 29-50.

Espada, J.P., Rosa, A. I., \& Méndez, F. X. (2003). Eficacia de los programas de prevención escolar con metodología interactiva. Salud y Drogas, 3 (2), 61-82.

Faggiano, F., Vigna-Taglianti, F., Burkhart, G., Bohrn, K., Cuomo, L., Gregori, D., ... \& van der Kreeft, P. (2010). The effectiveness of a school-based substance abuse prevention program: 18month follow-up of the EU-Dap cluster randomized controlled trial. Drug and alcohol dependence, 108(1), 56-64.

Faggiano, F., Vigna-Taglianti, F.D., Versino, E., Zambon, A., Borraccino, A., \& Lemma, P. (2008). School-based prevention for illicit 
drugs use: A systematic review. Preventive Medicine, 46, 385-396.

Fernández, S., Nebot, M., \& Jané, M., (2002) Evaluación de la efectividad de los programas escolares de prevención del consumo de tabaco, alcohol y cannabis: ¿Qué nos dicen los Meta-análisis? Revista Española de Salud Pública, 76 (3), 175-187.

Furr-Holden, C.D.M., Ialongo, N.S., Anthony, J.C., Petras, H., \& Kellam, S.G., (2004). Developmentally inspired drug prevention: middle school outcomes in a school-based randomized prevention trial. Drug and Alcohol Dependence, 73 (2), 149-158.

García, P., Fernández, A., Carrillo, A., \& Sánchez, J.M. (2006). Comparación de la eficacia de un programa de prevención del tabaquismo en la escuela desarrollado por personal sanitario con otro desarrollado por personal docente. Revista de Calidad Asistencial, 21 (3), 129-136.

García-Rodríguez, J.A., \& López-Sánchez, M.C. (1994). Cuestionario de consumo. En López Sánchez, C. (2000). Comparación de diversas técnicas Psicológicas en la Prevención de las Drogodependencias. (Tesis doctoral).

García-Rodríguez, J.A., \& López-Sánchez, C. (1998). Guía Técnica Barbacana I . Alicante: INID-UMH.

García-Rodríguez, J.A., \& López-Sánchez, C. (2001). Medida de las actitudes en la prevención de las drogodependencias: El alcohol. En J.A. García-Rodríguez y C. López-Sánchez (comps.) Manual de estudios sobre alcohol. (pp. 25-36). Madrid: EDAF.

García del Castillo, J.A., López-Sánchez, C., Fernández, I., \& Catalá, L. (2003). Evaluación del programa de prevención de drogas Barbacana. Salud y Drogas, 3 (1), 33-56.

Gázquez, M., García del Castillo, J.A., \& Espada, J.P. (2010). Eficacia de los programas de prevención escolar en función del agente preventivo: Profesores vs Expertos. Health and Addictions, 10 (2), 111-131.

Gómez, J. A., Luengo, M. A., \& Romero, E. (2002). Prevención del consumo de drogas en la escuela: cuatro años de seguimiento de un programa. Psicothema, 14 (4), 685-692.

Gottfredson, D.C., \& Wilson, D.B. (2003). Characteristics of effective school-based substance abuse prevention. Prevention Science, 4 (1), 27-38.

Guxens, M., Nebot, M., Ariza, C., \& Ochoa, D. (2007). Factores asociados al inicio del consumo de cannabis: una revisión sistemática de estudios de cohortes. Gaceta Sanitaria, 19 (3), 1-9.

Hansen, W. B., (1992). School-based substance abuse prevention: A review of the state of the art in curriculum: 1980-1990. Health Educational Research, 7 (3), 403-430.

Harrington, N.G., Giles, S.M., Hoyle, R.H., Feeney, G.J., \& Yungbluth, S.C. (2001). Evaluation of the all stars character education and problem behavior prevention program: Effects on mediator and outcome variables for middle school students. Health Education \& Behavior, 28 (5), $533-$ 546.

Kalant, H. (2004). Adverse effects of cannabis on health: an update of the literatura since 1996. Progress in Neuropsychopharmacology and Biological Psychiatry, 28 (5), 849-863.

Lemstra, M., Bennett, N., Nannapaneni, U., Neudorf, C., Warren, L., Kershaw, T., \& Scott, C. (2010). A systematic review of school-based marijuana and alcohol prevention programs targeting adolescents aged 10-15. Addiction Research \& Theory, 18 (1), 84-96.

Luengo, A., Gómez, J.A., Garra, A., Romero, E., \& Otero, J.M. (1998). Construyendo Salud. Promoción del desarrollo personal y social . Madrid: Ministerio de Educación y Cultura.

Luengo, A., Romero, E., Gómez-Fragüela, J.A., Guerra, A., \& Lence, P. (1999). 
Comparación de tres programas de prevención escolar para reducir el consumo de cannabis, en función del tipo de

La prevención del consumo de drogas y la conducta antisocial en la escuela: análisis y evaluación de un programa - Madrid: Ministerio de Educación y Cultura, Ministerio de Sanidad y Consumo y Ministerio del Interior.

Macià, D., Olivares, J., \& Méndez, F.X. (1993). Intervención comportamental-educativa en la prevención de la drogodependencia. En F.X. Méndez, D. Macià y J. Olivares, (Eds.) Intervención conductual en contextos comunitarios I: Programas aplicados de prevención (pp. 97-129). Madrid: Pirámide.

McBride, N., Farringdon, F., Midford, R., \& Phillips, M. (2001). SHAHRP final report to the Western Australian Health Promotion Foundation . National Drug Research Institute: Perth, Western Australia.

McNeal, R.B., Hansen, W.B., Harrington, N.G., \& Giles, S.M. (2004). How All Stars work: An examination of program effects on mediating variables. Health Education and Behavior, 31 (2), 165-178.

Mellanby, A., Rees, J., \& Tripp, J. (2000). Peerled and adult-led school health education: a critical review of available comparative research. Health Education Research, 15 , 533-545.

Merino, P.P. (2000). Vieja historia del cannabis y recientes prácticas preventivas en Europa. Adicciones, 12 (Supl.2), 275-280.

Moral, M.V., Rodríguez, F.J., Overjero, A., \& Sirvent, C. (2009). Cambios actitudinales y reducción de consumo de alcohol en adolescentes a partir de un programa de intervención psicosocial. Adicciones, 21 (3), 207-220.

Nozu, Y., Watanabe, M., Kubo, M., Sato, Y., Shibata, N., Uehara, C.,... Kito, H. (2006). Effectiveness of drug abuse prevention program focusing on social influences among high school students: 15month follow. Environmental Health and Preventive Medicine 11 , 75-81.

Observatorio Español sobre Drogas (2010). Informe 2009. Situación y tendencias de los problemas de drogas en España. Madrid: Delegación del Gobierno para el Plan Nacional sobre Drogas (DGPNSD). Ministerio de Sanidad y Política Social: OED.

Observatorio Europeo de las Drogas y Toxicomanías (2009). Informe Anual 2010: el problema de la drogodependencia en Europa . Observatorio Europeo de las Drogas y las Toxicomanías, Lisboa: OEDT.

Oficina de las Naciones Unidas contra la Droga y el Delito. UNODC (2010). Informe Mundial sobre las drogas . Nueva York: Publicación de las Naciones Unidas.

Parolaro, D. (2010). Consumo de cánnabis de los adolescentes y esquizofrenia: evidencias epidemiológicas y experimentales. Adicciones, 22 (3), 185-190.

Pereira, J.R., \& García-Fernández, J.M. (2009). Evaluación de los efectos del programa preventivo Saluda según la edad de los participantes. Health and Addictions, 9 (1), 93-111.

Rohrbach, L.A., Dent, C.W., Skara, S., Sun, P., \& Sussman, S. (2007). Fidelity of Implementation in Project Towards No Drug Abuse (TND): A comparison of Classroom Teachers and Program Specialists. Prevention Science, 8 , 125-132.

Sánchez-Meca, J., Marín-Martínez, F., \& Chacón-Moscoso, S. (2003). Effects-size indices for dichotomized outcomes in meta-analysis. Psychological Methods, 8 , 448-467.

Shope, J., Elliott, M., Raghunathan, T., \& Waller, P. (2001). Long term followup of a high school Alcohol Misuse Prevention Programme's effect on students subsequent driving. Alcoholism: Clinical and Experimental Research, 25 (3), 403-410.

Skara, S., Rohrbach, L.A., Sung, P., \& Sussman, S. (2005). An evaluation of the fidelity of implementation of a school-based program: 
Project Toward no Drug Abuse (TND). Journal Drug Education, 35 (4), 305-329.

Skara, S., \& Sussman S. (2003). A review of 25 long-term adolescent tobacco and other drug use prevention program evaluations. Preventive Medicine, 37 , 451-474.

St. Pierre, T. L., Osgood, D. W, Siennick, S.E, Kauh, T.J., \& Burden, F. F. (2007). Project ALERT whit Outside Leaders: What Leader Characteristics are important for Success? Prevention Science, 8, 51- 64.

Stead, M., \& Angus, K. (2004). Literature review into the effectiveness of school drug education . Edinburgh: Institute for Social Marketing, University of Stirling.

Sun, P., Sussman, S., Dent, C.W., \& Rohrbach, L.A. (2008). One-year followup evaluation of Project Towards No Drug Abuse (TND-4). Preventive Medicine, 47 , 438-442.

Sussman, S., Dent, C.W., Craig, S., RittOlsen, A., \& McCuller, W.J., (2002). Development and immediate impact of a self-instruction curriculum for an adolescent indicated drug abuse prevention trial. Journal of Drug Education, 32 (2), 121-137.

Sussman, S., Sun, P., McCuller, W.J., \& Dent, C.W., (2003). Project Towards No Drug Abuse: two-year outcomes of a trial that compares health educator delivery to selfinstruction. Preventive Medicine, 37 (2), 155-162.

Tobler, N.S., Roona, M.R., Ochshorn, P., Marshall, D.G., Streke, A.V., \& Stackpole, K.M. (2000). School-based adolescent drug prevention programs: 1998 meta-analysis. Journal of Primary Prevention, 20 (4), 275-336.

White, D., Buckley, E., \& Hassan, J. (2004). Literature Review on the Role of External Contributors in School Drug, Alcohol and Tobacco Education. Research Report RR514. Staffordshire: Centre for Health Psychology.

\section{Notas}

* Artículo de investigación. El estudio descrito en este artículo forma parte de un proyecto de investigación financiado por la Convocatoria de Ayudas a la Investigación 2007 (Instituto de Cultura Juan Gil Albert. Diputación Provincial de Alicante, España). Agradecimientos: El estudio descrito en este artículo forma parte de un proyecto de investigación financiado por la Convocatoria de Ayudas a la Investigación 2007 (Instituto de Cultura Juan Gil Albert. Diputación Provincial de Alicante, España). Conflicto de intereses: Los autores declaran que no tienen conflictos de intereses. Los autores son los únicos responsables del contenido y la redacción del documento. 